\title{
Testing Maximal 1-Planarity of Graphs with a Rotation System in Linear Time ${ }^{\star}$ (Extended Abstract)
}

\author{
Peter Eades $^{1}$, Seok-Hee Hong ${ }^{1}$, Naoki Katoh ${ }^{2}$, Giuseppe Liotta ${ }^{3}$, \\ Pascal Schweitzer ${ }^{4}$, and Yusuke Suzuki ${ }^{5}$ \\ 1 University of Sydney, Australia \\ \{peter.eades, seokhee.hong\}asydney.edu.au \\ 2 Kyoto University, Japan \\ naoki@archi.kyoto-u.ac.jp \\ ${ }^{3}$ Universitá di Perugia, Italy \\ liotta@diei.unipg.it \\ 4 Australian National University, Australia \\ pascal.schweitzer@anu.edu.au \\ ${ }^{5}$ Niigata University, Japan \\ y-suzuki@math.sc.niigata-u.ac.jp
}

\begin{abstract}
A 1-planar graph is a graph that can be embedded in the plane with at most one crossing per edge. It is known that testing 1-planarity of a graph is NP-complete. A 1-planar embedding of a graph $G$ is maximal if no edge can be added without violating the 1-planarity of $G$. In this paper we show that the problem of testing maximal 1-planarity of a graph $G$ can be solved in linear time, if a rotation system (i.e., the circular ordering of edges for each vertex) is given. We also prove that there is at most one maximal 1-planar embedding of $G$ that preserves the given rotation system, and our algorithm produces such an embedding in linear time, if it exists.
\end{abstract}

\section{Introduction}

A recent research topic in topological graph theory is the study of graphs that are almost planar in some sense. Examples of such almost planar graphs are 1-planar graphs, which can be embedded in the plane with at most one crossing per edge.

The class of 1-planar graphs was first considered by Ringel [10] and subsequently has been extensively investigated; see, for example, [2 5|7]. In particular, Pach and Toth proved that a 1-planar graph with $n$ vertices has at most $4 n-8$ edges [9].

Testing 1-planarity of a graph is NP-complete [8]. This holds even if the input graph is near-planar (i.e., deletion of an edge makes the resulting graph planar) [3], and even

\footnotetext{
* In this extended abstract, proofs are omitted. For the full version of this paper, see [4]. This work was initiated at the Workshop on Geometric Graph Theory 2011, June, Port Douglas, Australia, organized by Peter Eades and Seok-Hee Hong, and funded by the University of Sydney. Peter Eades and Seok-Hee Hong are partially supported by the Australian Research Council. Giuseppe Liotta is partially supported by MIUR of Italy under project Algo-DEEP prot. 2008TFBWL4; Pascal Schweitzer is supported by the National Research Fund, Luxembourg and co-funded under the Marie Curie Actions of the European Commission FP7-COFUND.
} 
if the rotation system (i.e., the circular ordering of edges for each vertex) is given as part of the input [1].

A 1-planar embedding of a graph $G$ is maximal if no edge can be added without violating the 1-planarity of $G$ [11]12]. A maximal 1-plane graph $G_{\xi}$ is a graph embedded in a plane with a maximal 1 -planar embedding $\xi$.

In this paper, we investigate the problem of testing whether a graph with a given rotation system is maximal 1-planar. We give a linear time algorithm. To obtain linear time, we prove various combinatorial properties of maximal 1-plane graphs. We first show that in any maximal 1-planar embedding, the subgraph induced by the edges that do not intersect any other edge (also called the "red graph") is spanning and biconnected. We show that if the rotation system does admit an embedding, then it is unique. Our algorithm also produces a maximal 1-planar embedding $\xi$ of $G$ consistent with $\Phi$ in linear time, if it exists.

\section{Preliminaries}

A drawing $D(G)$ of a graph $G$ is a geometric representation of a graph in the plane, such that each vertex of $G$ is mapped to a point in the plane, and each edge of $G$ is drawn as a curve. As customary, in this paper we only allow proper drawings: no two edges incident to a vertex cross each other; two edges cross at most once; edges may not touch each other tangentially; no three edges cross at a single point.

A drawing $D(G)$ of a graph $G$ induces an embedding $\xi(G)$ of a graph $G$. More precisely, an embedding $\xi(G)$ of a graph $G$ is defined by a circular ordering of the edges around each vertex in $G$, as well as the combinatorial configuration of the crossings in a drawing $D(G)$. We say that the drawing $D(G)$ represents $\xi(G)$.

An embedding $\xi(G)$ is called planar, if there is no crossing in any drawing $D(G)$ representing $\xi(G)$. A graph $G$ is called planar, if it admits a planar embedding. A plane graph $G_{\xi}$ is a graph $G$ embedded in a plane with a planar embedding $\xi$. An embedding $\xi(G)$ is called 1-planar, if each edge crosses at most one other edge in a drawing $D(G)$ representing $\xi(G)$. A graph $G$ is called 1-planar, if it admits an 1-planar embedding. A 1-plane graph $G_{\xi}$ is a graph $G$ embedded in a plane with a 1-planar embedding $\xi$.

Given a 1-planar embedding $\xi(G)$ of a 1-planar graph $G$, we can obtain a planar embedding by replacing each crossing in $\xi(G)$ with a virtual vertex. We call the resulting planar graph the planarization of $G$, and denote it by $G_{P}$. Note that $\xi\left(G_{P}\right)$ preserves $\xi(G)$. The original vertices of $G$ are called real vertices. The planarization of $G$ uniquely determines the embedding $\xi(G)$.

A 1-planar embedding $\xi(G)$ is called maximal, if no edge can be added without violating 1-planarity of $G$ [11|12]. A maximal 1-plane graph $G_{\xi}$ is a graph embedded in a plane with a maximal 1-planar embedding $\xi$.

A graph $G$ is maximal 1-planar if addition of any edge destroy 1-planarity of $G$ [12]. In contrast to this, a maximum 1-planar graph is a graph with a maximal number of edges among all 1-planar graphs on the same number of vertices [12].

A rotation system $\Phi(G)$ of a graph $G$ defines a circular ordering of edges around each vertex in $G$. Without loss of generality, we assume that the circular ordering is clockwise, unless otherwise stated. Let $G_{\Phi}$ denote a graph $G$ with a given rotation system 
$\Phi$. If an embedding $\xi(G)$ of a graph $G$ induces a rotation system $\Phi$, we say that $\xi(G)$ represents $\Phi$ or that $\xi(G)$ is consistent with $\Phi$.

Note that a rotation system $\Phi$ does not define crossings between edges. Thus, in general, there may be several 1-planar embeddings of a graph representing a given rotation system. However, for a planar graph, a rotation system uniquely determines a planar embedding (i.e., there is at most one planar embedding representing a given rotation system). Thus, to determine a 1-planar embedding, it is sufficient to determine a rotation system of the planarization of the 1-planar embedding.

An embedding $\xi(G)$ of a graph $G$ in the plane defines the crossing-free edges as well as the crossing edges. We call the crossing-free edges red edges, and call the edges that are crossed blue edges. The red graph $G_{R}$ of a graph $G$ is the subgraph induced by the red edges.

When we define a subgraph $G^{\prime}$ of a graph $G$, we assume that $G^{\prime}$ inherits the topology of $G$. For example, if $G$ is an embedded graph, then we treat the subgraph $G^{\prime}$ of $G$ as an embedded graph, preserving the embedding of $G$ (i.e., $\xi\left(G^{\prime}\right)$ is induced by $\xi(G)$ ). Similarly, if $G$ has a rotation system $\Phi(G)$, then the subgraph $G^{\prime}$ of $G$ inherits the rotation system of $G$ (i.e., $\Phi\left(G^{\prime}\right)$ is induced by $\Phi(G)$ ).

\section{Properties of Maximal 1-Planar Embeddings}

This section presents combinatorial properties of maximal 1-planar embeddings. We first present two technical Lemmas.

Lemma 1. Let $\xi(G)$ be a 1-planar embedding of a 1-planar graph $G$ on at least 2 vertices, and $G_{P}$ be the planarization of $G$. Each face $f$ of $G_{P}$ has at least two real vertices.

Lemma 2. Let $G$ be a maximal 1-plane graph. If $v_{1}$ and $v_{2}$ are consecutive neighbors of a virtual vertex $w$ in the planarization $G_{P}$ of $G$, then there are two internally vertex disjoint red paths (that is, paths consisting of red edges) from $v_{1}$ to $v_{2}$.

These technical Lemmas lead to the main structural Theorem, that the red subgraph of a maximal 1-planar embedding is biconnected and spanning.

Theorem 1. In a maximal 1-planar embedding $\xi(G)$ of a graph $G$ with at least 3 vertices, the red graph $G_{R}$ is biconnected and spans all vertices.

In the following section, we use Theorem 1 to design a linear time maximal 1-planarity testing algorithm for a graph $G$ with a given rotation system.

\section{Testing Maximal 1-Planarity of a Graph with a Rotation System}

In this section, we sketch a linear time algorithm to test maximal 1-planarity of a graph with a given rotation system. More precisely, we prove the following theorem. 
Theorem 2. There exists a linear time algorithm that tests whether graph $G$ with a given rotation system $\Phi$ has a maximal 1-planar embedding representing $\Phi$. In case such an embedding exists, it is unique and the algorithm computes this embedding.

An overview of the maximal 1-planarity testing algorithm consists of five steps, as in Algorithm 11. Steps 1 and 3 use the Pach-Toth bound [9], a standard biconnectivity algorithm, and a standard planarity algorithm. In the following subsections, we sketch Steps 2, 4 and 5.

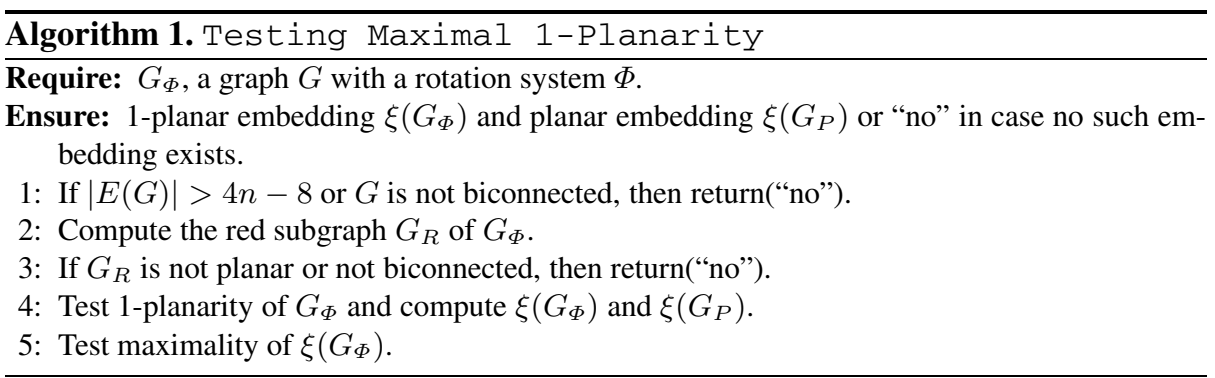

\subsection{Computing the Red Subgraph $G_{R}$}

It is convenient to regard the input graph $G$ as a directed graph, with two directed edges $(u, v)$ and $(v, u)$ for each pair $u, v$ of adjacent vertices. We say that a directed edge $\left(v_{2}, v_{3}\right)$ is the rightmost continuation of a directed edge $\left(v_{1}, v_{2}\right)$, if the vertex $v_{3}$ is the vertex that precedes $v_{1}$ in the circular ordering of $v_{2}$.

We say that a walk $v_{1}, \ldots, v_{t}$ is a completed rightmost walk, if the following two conditions hold (where indices are taken modulo $t$ ): (1) for every $i \in\{1, \ldots, t\}$, the directed edge $\left(v_{i}, v_{i+1}\right)$ is the rightmost continuation of the directed edge $\left(v_{i-1}, v_{i}\right)$, and (2) for all $i, j \in\{1, \ldots, t\}$, if $\left(v_{i}, v_{i+1}\right)=\left(v_{j}, v_{j+1}\right)$, then $i=j$.

Completed rightmost walks characterize the colors of the edges of $G_{\Phi}$.

Lemma 3. Let $G$ be a 1-plane graph with a given rotation system $\Phi$, whose red graph $G_{R}$ is spanning and biconnected. An edge e of $G$ is red if and only if there is a completed rightmost walk on $G_{\Phi}$ that traverses e only in one direction.

Lemma 3 implies that the color of an edge is invariant over all 1-planar embeddings that represent $G_{\Phi}$ and have a red graph $G_{R}$ that is spanning and biconnected. Thus we can define algorithm that takes $G_{\Phi}$, a graph $G$ with a rotation system $\Phi$, as input, and computes the color of the edges in such embeddings. The algorithm simply traverses the graph with rightmost walks. Using a system to mark edges after they have been traversed, one can color the edges red or blue.

Lemma 4. Let $G_{\Phi}$ be a graph $G$ with a rotation system $\Phi$. There is a linear time algorithm that computes a coloring of the edges of $G_{\Phi}$ into red and blue. This coloring is equal to the coloring in every 1-planar embedding that represents $G_{\Phi}$ with a spanning and biconnected red graph. 


\subsection{Computing a 1-Planar Embedding of $G_{\Phi}$}

Given a rotation system $\Phi$, and a coloring of the edges of $G$ into red and blue such that the red graph is spanning and biconnected, we now test whether there exists a 1-planar embedding of $G_{\Phi}$ consistent with the colors. If such an embedding exists, we compute a planar embedding of the planarization $G_{P}$ of $G_{\Phi}$.

After testing biconnectivity and planarity of $G_{R}$ in Step 3 of Algorithm 1, we have a planar embedding $\xi\left(G_{R}\right)$ of $G_{R}$ which preserves the given rotation system $\Phi$ of $G$. This embedding is used as input for Step 4 of Algorithm 1 .

The following lemma shows that the 1-planar embedding of $G_{\Phi}$ is unique, if it exists.

Lemma 5. A graph $G$ with a given rotation system $\Phi$ can be represented by at most one 1-planar embedding consistent with $\Phi$ for which the red graph $G_{R}$ is spanning and biconnected.

Using Lemma[5, we can test 1-planarity of $G_{\Phi}$ in linear time under the assumption that $G_{R}$ is spanning and biconnected.

Lemma 6. Let $G$ be a graph with a rotation system $\Phi$, and suppose that $G_{R}$ is a planar, spanning and biconnected subgraph of $G$. There exists a linear time algorithm that tests whether there is a 1-planar embedding of $G_{\Phi}$ that is consistent with $\Phi$ such that $G_{R}$ is the red subgraph. In case such an embedding $\xi\left(G_{\Phi}\right)$ exists, it is unique and the algorithm also produces the planar embedding $\xi\left(G_{P}\right)$ of the planarization of $\xi\left(G_{\Phi}\right)$.

Sketch of proof: Lemma 5 implies that we can use the rotation system $\Phi$ to identify the red facial cycles and the blue edges inside each red face. Then crossings can be detected by traversing each red face; this traversal identifies any edge with more than one crossing.

\subsection{Testing Maximality of 1-Planar Embedding}

In this section, we show that maximality of a 1-planar embedding of a graph $G$ with a given rotation system $\Phi$ can be tested in linear time. We first present some properties of maximal 1-planar graphs.

Lemma 7. Suppose that $\xi(G)$ is a maximal 1-planar embedding of a graph $G$. If two vertices $v_{1}$ and $v_{2}$ of $G$ are incident with the same face $f$ in the planarization $G_{P}$ of $\xi(G)$, then $v_{1}$ and $v_{2}$ are adjacent in $G$.

Lemma 8. Let $\xi(G)$ be a maximal 1-planar embedding of a graph $G$. Let $G_{P}$ be the planarization of $G$, and $\xi\left(G_{P}\right)$ be the planar embedding of $G_{P}$ induced by $\xi(G)$. Let $f$ be a facial cycle in $\xi\left(G_{P}\right)$.

(a) Each crossing in $\xi(G)$ induces a 4-clique.

(b) The face $f$ has at most 4 real vertices.

(c) The face $f$ has at most 8 vertices (real plus virtual). 
We now sketch a linear time algorithm that tests whether a 1-planar embedding is maximal. We can simply test whether there are two nonadjacent real vertices $v_{1}$ and $v_{2}$ such that adding the edge $\left(v_{1}, v_{2}\right)$ does not destroy 1-planarity. We first check each face to check whether it contains two such $v_{1}$ and $v_{2}$. Then we test red edge $e$ to see whether there are a pair $v_{1}$ and $v_{2}$ on the faces separated by $e$ to see whether one can add $\left(v_{1}, v_{2}\right)$ by crossing $e$. Using Lemmas 7 and 8 , one can perform these checks in linear time.

Lemma 9. Let $\xi\left(G_{\Phi}\right)$ be a 1-planar embedding of $G_{\Phi}$, and $\xi\left(G_{P}\right)$ be a planar embedding of $G_{P}$, the planarization of $G_{\Phi}$. There is a linear time algorithm to test whether $G_{\Phi}$ is maximal 1-planar.

The correctness of steps 2, 4, and 5 of Algorithm 1 follow from Lemmas 4, 6, 9 respectively. Theorem 2 follows.

\section{Conclusion}

This paper presents a linear time algorithm that takes a graph $G$ with a rotation system $\Phi$ as input, and tests whether $G$ has a maximal 1-planar embedding of $G$ consistent with $\Phi$. The algorithm constructs the embedding if it exists.

The existence of a 1-planar embedding does not guarantee the existence of a straightline 1-planar drawing [6|13]. However, there is a linear time algorithm to construct such a drawing if it exists; see [6].

For future work, it would be interesting to know whether the problem of testing maximal 1-planarity is NP-hard, without the assumption that the rotation system is given. Further, we would like to consider similar algorithmic questions for $k$-planar graphs, that is, embeddings with at most $k$ crossings per edge. Testing $k$-planarity is NP-hard (see [8]), but perhaps the testing problem could be easier if a rotation system was part of the input or if maximality was assumed.

\section{References}

1. Auer, C., Brandenburg, F.J., Gleißner, A., Reislhuber, J.: On 1-planar graphs with rotation systems. Tech. Rep. MIP1207, Faculty of Informatics and Mathematics, University of Passau (2012)

2. Borodin, O.V.: Solution of the Ringel problem on vertex-face coloring of planar graphs and coloring of 1-planar graphs. Metody Diskret. Analiz. 41, 12-26, 108 (1984)

3. Cabello, S., Mohar, B.: Adding one edge to planar graphs makes crossing number and 1planarity hard. CoRR abs/1203.5944 (2012)

4. Eades, P., Hong, S.H., Katoh, N., Liotta, G., Schweitzer, P., Suzuki, Y.: Testing maximal 1planarity of graphs with a rotation system in linear time. TR IT-IVG-2012-02, School of IT, University of Sydney (2012)

5. Eades, P., Liotta, G.: Right Angle Crossing Graphs and 1-Planarity. In: van Kreveld, M., Speckmann, B. (eds.) GD 2011. LNCS, vol. 7034, pp. 148-153. Springer, Heidelberg (2012)

6. Hong, S.-H., Eades, P., Liotta, G., Poon, S.-H.: Fáry's Theorem for 1-Planar Graphs. In: Gudmundsson, J., Mestre, J., Viglas, T. (eds.) COCOON 2012. LNCS, vol. 7434, pp. 335346. Springer, Heidelberg (2012) 
7. Hudák, D., Madaras, T.: On local properties of 1-planar graphs with high minimum degree. Ars Math. Contemp. 4(2), 245-254 (2011)

8. Korzhik, V.P., Mohar, B.: Minimal obstructions for 1-immersions and hardness of 1-planarity testing. J. Graph Theory (2012)

9. Pach, J., Tóth, G.: Graphs drawn with few crossings per edge. Combinatorica 17(3), 427-439 (1997)

10. Ringel, G.: Ein Sechsfarbenproblem auf der Kugel. Abh. Math. Sem. Univ. Hamburg 29, 107-117 (1965)

11. Suzuki, Y.: Optimal 1-planar graphs which triangulate other surfaces. Discrete Mathematics 310(1), 6-11 (2010)

12. Suzuki, Y.: Re-embeddings of maximum 1-planar graphs. SIAM J. Discrete Math. 24(4), $1527-1540$ (2010)

13. Thomassen, C.: Rectilinear drawings of graphs. Journal of Graph Theory 12(3), 335-341 (1988) 